\section{Dermatitis herpetiformis in an adolescent patient $^{*}$}

\author{
Adelina Costin ${ }^{1}$ \\ Ana Marta António ${ }^{1}$ \\ Constança Furtado ${ }^{1}$ \\ Elvira Bártolo ${ }^{1}$
}

DOI: http:/ / dx.doi.org/10.1590/abd1806-4841.20198227

\section{Dear Editor,}

Dermatitis herpetiformis is a pruritic, blistering skin disease affecting the extensor surface of the limbs, the buttocks and lower back. The autoantigen involved is transglutaminase-3, an enzyme of epidermis. ${ }^{1}$ The diagnosis is confirmed by direct immunofluorescence of perilesional skin, with a pathognomonic pattern of granular IgA deposits in papillary dermis, with a false-negative rate of $5 \%$.

Dermatitis herpetiformis and coeliac disease, both on the gluten intolerance spectrum, share immunomediation by IgA autoantibodies. About $75 \%$ of dermatitis herpetiformis patients show villous atrophy, identical to that observed in coeliac disease. ${ }^{2}$ Further supporting evidence includes, namely, the resolution of skin lesions after starting gluten-free diet, even if small bowel mucosa is normal; the detection of circulating transglutaminase- 3 antibodies in both patient populations; and the finding of subclinical inflammation of small bowel mucosa in dermatitis herpetiformis patients, convincingly link the two conditions. We can therefore state that dermatitis herpetiformis is not just an associated condition, like some autoimmune diseases, but is in fact the cutaneous manifestation of coeliac disease. ${ }^{3}$

A 17-year-old adolescent, with no past medical history, presented to our department with itchy papulovesicles and excoriations on the elbows, knees and buttocks, occurring for the past 3 months (Figures 1 and 2). He denied any abdominal symptoms, weight loss, delayed puberty, chronic fatigue or symptoms attributable to aphthous stomatitis. No family history of gastrointestinal

Received 25 February 2017

Accepted 09 August 2018.

* Study conducted at the Hospital Garcia de Orta, Almada, Portugal. Financial support: None.

Conflict of interest: None.

1 Dermatology and Venereology Service, Hospital Garcia de Orta, Almada, Portugal.

MAILING AdDREsS:

Adelina Costin

E-mail: adelina_costin@hotmail.com

(C)2019 by Anais Brasileiros de Dermatologia disease in first degree relatives was elicited. He had previously been treated with oral antibiotics, several antifungal creams and topical corticosteroids. Initial laboratory analyses were normal, including complete blood count, thyroid function and serum immunofixation and liver biochemistry. At the first visit, a skin biopsy was performed, and skin scrapings were obtained to test of fungal infection, as the main differential was tinea incognito secondary to topical corticosteroids use.

The fungal culture isolated Microsporum audouinii, so a trial of oral itraconazole and benzyl benzoate lotion was administered. The patient reported a brief improvement of the skin lesions and the pruritus. A second skin biopsy was obtained for histopathologic and direct immunofluorescence examinations, documenting neutrophilic abscesses in the dermal papillae and granular deposition of IgA within the dermal papillae of the perilesional skin specimen, confirming the main differential diagnosis of dermatitis herpetiformis at this stage (Figure 3).

Further investigation documented positivity for IgA antibodies to endomysium and tissue transglutaminase. Genetic analysis of peripheral blood sample confirmed the HLA-DQ2 haplotype. The patient was started on gluten-free diet and oral dapsone, after

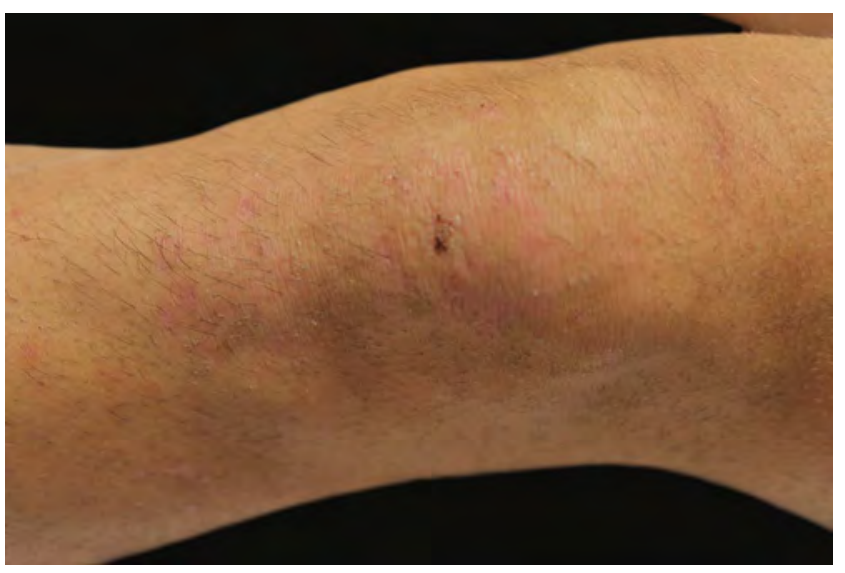

Figure 1: Dermatitis herpetiformis. Papules, vesicles and excoriations on the knee

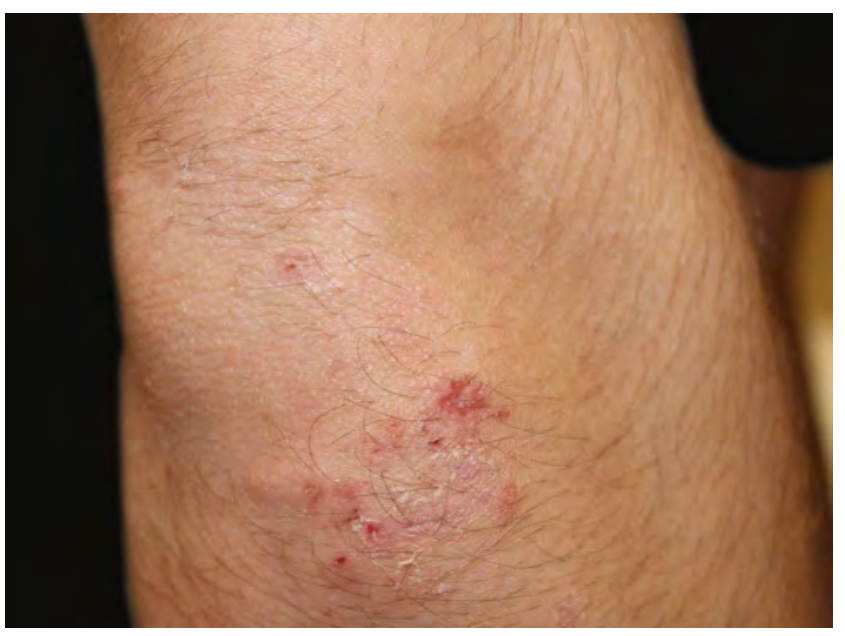

Figure 2: Dermatitis herpetiformis. Papules, vesicles and excoriations on the elbow 


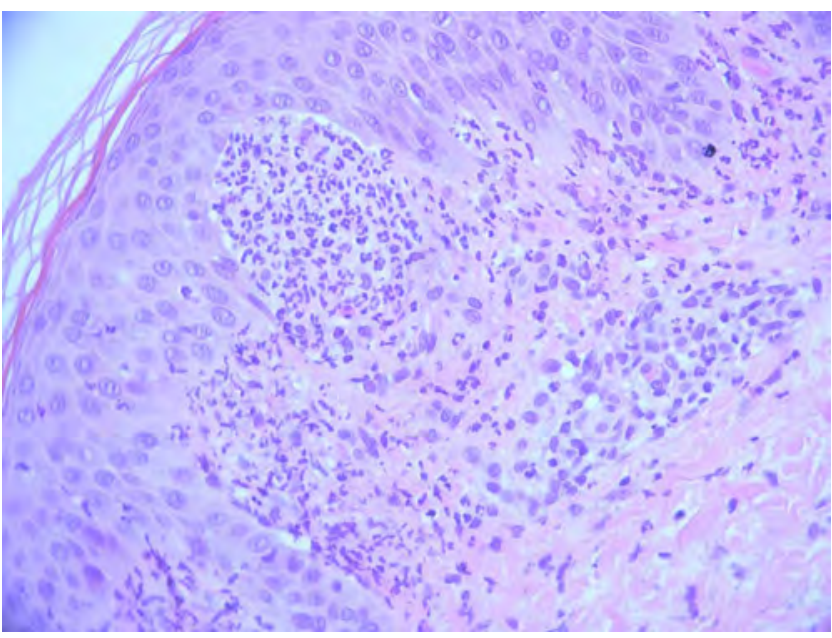

FIGURE 3: Small neutrophilic abscesses in dermal papillae and dermo-epidermal clefts overlying the abscesses. (Hematoxylin \& eosin, x100)

determining the levels of glucose-6-phosphate dehydrogenase, with a steady improvement in pruritus and regression of the cutaneous lesions after several weeks of treatment.

Dermatitis herpetiformis in childhood seems to be rare, with only $4 \%$ reported in a Finnish study. ${ }^{4}$

Although dermatitis herpetiformis is regarded as the cutaneous manifestation of gluten hypersensitivity spectrum, most patients either do not report it or report only minor gastrointestinal symptoms; in fact, iron deficiency anemia or pernicious anemia could constitute the only manifestation of coeliac disease in these patients.

The diagnostic algorithm for coeliac disease in children and adolescents varies according to symptoms or signs suggestive of coeliac disease, namely, chronic diarrhea, failure to thrive unexplained by other causes, weight loss, delayed puberty, nausea and/ or vomiting, amenorrhea, abdominal cramping and/or pain, chronic constipation, fatigue, recurrent aphthous stomatitis, fractures with minimal trauma, osteopenia/osteoporosis and liver chemistry abnormalities.

The diagnosis in these patients may be established without performing an intestinal biopsy if first-line serologic marker levels (e.g., IgA to tissue transglutaminase, total IgA and IgG to gliadine) are sufficiently elevated. ${ }^{5}$ If the IgA antibodies to tissue transglutaminase are at least 10x the upper normal level, one can proceed to detect IgA class antibodies to endomysium and perform a molecular test for coeliac disease (identifying one of the haplotypes associated with coeliac disease, HLA-DQ2 or DQ8); if both are positive, then the diagnosis is confirmed.

Virtually all patients with coeliac disease or dermatitis herpetiformis have one of these two haplotypes. Its importance derives from the fact that negative results could exclude the diagnosis.

Gluten avoidance is essential for the healing of intestinal mucosa and symptom relief in coeliac disease patients. While the gastrointestinal symptoms take a few weeks to disappear, the itchy skin lesions in dermatitis herpetiformis could take months or years to regress. Consequently, most patients are also treated with dap- sone, which is useful in relieving dermatological symptoms but has no effect on gastrointestinal inflammation.

This report emphasizes the need for a careful workup and a high degree of suspicion when evaluating itchy dermatosis in children or adolescents with no gastrointestinal symptoms.]

\section{REFERENCES}

1. Sárdy $M$, Kárpáti $S$, Merkl $B$, Paulsson $M$, Smyth N. Epidermal transglutaminase (TGase 3) is the autoantigen of dermatitis herpetiformis. J Exp Med. 2002;195:747-57.

2. Marks J, Shuster S, Watson AJ. Small $\neg$ bowel changes in dermatitis herpetiformis. Lancet. 1966;2:1280-2.

3. Savilahti E, Reunala T, Mäki M. Increase of lymphocytes bearing the gamma/delta T cell receptor in the jejunum of patients with dermatitis herpetiformis. Gut. 1992;33:206-11.

4. Hervonen K, Salmi TT, Kurppa K, Kaukinen K, Collin P, Reunala T. Dermatitis herpetiformis in children: a long-term follow-up study. $\mathrm{Br}$ J Dermatol. 2014;171:1242-3.

5. Husby S, Koletzko S, Korponay-Szabó IR, Mearin ML, Phillips A, Shamir R, et al. European Society for Paediatric Gastroenterology, Hepatology, and Nutrition guidelines for the diagnosis of coeliac disease. J Pediatr Gastroenterol Nutr. 2012;54:136-60.

Adelina Costin
Approval of the final version of the manuscript; Conception and planning
of the study; Elaboration and writing of the manuscript; Effective participa-
tion in research orientation, Critical review of the literature, Critical review
of the manuscript.

Ana Marta António D ORCID 0000-0002-2495-0348

Approval of the final version of the manuscript; Conception and planning of the study; Critical review of the manuscript.

Constança Furtado (D) ORCID 0000-0002-7127-766X Approval of the final version of the manuscript; Conception and planning of the study; Intellectual participation in propaedeutic and/or therapeutic conduct of the cases studied; Critical review of the manuscript.

Elvira Bártolo

$$
\text { (1) ORCID }
$$

0000-0002-8342-2836

Approval of the final version of the manuscript; Intellectual participation in propaedeutic and/or therapeutic conduct of the cases studied; Critical review of the manuscript.

How to cite this article: Costin A, António AM, Furtado C, Bártolo E. Dermatitis herpetiformis in an adolescent patient. An Bras Dermatol. 2019;94(4):495-6. 